Journal of Engineering and Applied Sciences 14 (10): 3354-3358, 2019

ISSN: 1816-949X

(C) Medwell Journals, 2019

\title{
Proposed Medical Cloud Computing for Iraqi Health Institutions
}

\author{
${ }^{1}$ Thsan Ali Hassan, ${ }^{2}$ Ahmed Adnan Mohammed and ${ }^{3}$ Nibras Othman Abdulwahid \\ ${ }^{1}$ College of Medicine, University of Diyala, Baqubah, Iraq \\ ${ }^{2}$ University of Diyala, Baqubah, Iraq \\ ${ }^{3}$ Ministry of Higher Education and Scientific Research, Baghdad, Iraq
}

\begin{abstract}
In the last few years, the interest in information technology in all aspects of life in Iraq has begun. Especially, health care because it is important to create a healthy generation. The systems currently used to collect vital data for patients require high effort and time as well as the possibility of errors in the provision of treatment to patients, all of these prevents access to patient data in a real-time. In this study, we presented a proposal for a system based on cloud computing technology. The medical staff needs only an internet-connected device with a browser to access patient data at any time and from anywhere. Therefore, it provides the possibility of telemedicine service. In addition, this proposed system reduces medical errors, eliminates the collection of manual data and finally, helps the doctor to make decisions quickly and accurately. Which is reflected in the provision of treatment of the patient in a real-time and with high efficiency which is the main objective of this proposed system.
\end{abstract}

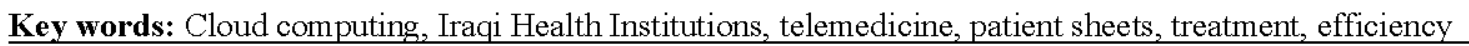

\section{INTRODUCTION}

Cloud Computing $(\mathrm{CC})$ allows the information to become accessible to everyone in a way that could not be imagined by some. In our time, I see it being called the "cloud era" where all the digital information in the whole world is stored inside the electronic cloud which enables anyone with any digital device and internet access to get the information they want. As well, cloud computing has many features such as safety, reliability, scalability and high flexibility (Mei and Qiu, 2016). In recent years, cloud computing has played an important role in all areas of education and business. It is also possible to play the same role in the health sector. Cloud computing is defined according to the National Institute of Standards and Technology (NIST) as "a model for enabling convenient, on demand network access to a shared pool of configurable computing resources that can be rapidly provisioned and released with minimal management effort or service provider's interaction which are not normally provided in the regular health care environments" (Bamiah et al., 2012; Aziz and Guled, 2016).

$\mathrm{CC}$ has evolved as a new model of information technology provides a graceful way to deliver health care services in a smart way. On the other hand, cloud computing has enabled telemedicine that allows remote diagnosis and surveillance of patients (Rolim et al., 2010). In the Iraqi Health Institutions (IHI), there are huge amounts of data collected and recorded from patients daily, these data are important and vital of making the decision by doctors to provide appropriate treatment for these patients in real-time. Therefore, we need unconventional ways to save this data, ensuring accuracy, speed, easy access and reduce the effort required to save these data, one of these methods is cloud technologies (Tejaswi et al., 2012). Cloud computing has the following essential characteristics:

Broad access to the network: Wide range of network access capabilities through many client platforms (Wang and Alexander, 2013)

Quick flexibility: Capacity can be flexible, fast and automatic in some cases.

On-demand self-service: The cloud user can access the possibilities of computing from one side such as network storage and server time automatically without the need for interaction by the cloud service provider (Doukas et al., 2010).

Pooling of resources: The computing capabilities of the provider are combined to serve many consumers by using a multi-tenant model (Daman et al., 2016).

Literature review: The use of cloud technology in the medical field is begun to take on a wide scale with solutions to the problems facing the medical staff in how to deal with big data for patients. So, there are many works that discussed employing the cloud computing in the medical field.

Corresponding Author: Ihsan Ali Hassan, College of Medicine, University of Diyala, Baqubah, Iraq 
The research by Huang et al. (2011) provide a solution for how to manage and analyze medical data with high efficiency. This solution relies on cloud computing to provide high storage resources and accessibility where it is proposed to use a platform called Hadoop to solve problems of sharing, storage and exchange of medical images.

Kanagaraj and Sumathi (2011) proposed a system based on the concept of cloud computing where it is possible to share and exchange medical information and advanced processing in the cloud. Medical images (information) in the cloud meet the necessary details needed by the doctors and patients can seek treatment at different branches of the hospital. Also, reducing the need to maintain computer resources in the hospital. In addition, medical equipment will be rebuilt to be more efficient and cost-effective as medical terminal units.

\section{MATERIALS AND METHODS}

Motivation: The system in the Iraqi Health Institutions is a manual system. When the patient enters the hospital, the medical staff records the patient's data on pre-prepared study manually and this studies called "Patient Sheets", then enter this data to computers by using special systems and programs. Moreover, this data is stored into local servers as database for organizing and make it accessed through friendly interface. Figure 1 illustrates the process of writing patient's data as follow:

- The doctor write patient's data

- The medical staff enters the data into the computer

- Data is stored in a local servers

Medical staff can access patient's information through interface applications. This system has a set of problems that affect the process of treating the patient:

- The process is slow and arduous

- The possibility of errors where data entry for computers depends on what the doctor writes and some doctors their handwriting is incomprehensible

- The time required to collect patient data and provide it to the medical staff, causing a delay in research

If we follow the steps of writing patient data from the time of admission to the hospital to the possibility of retrieving these data, there is a lost time between those steps. This time causes non-monitoring of the patient's condition in real-time, causing delayed treatment of the patient. So, we suggested a solution that would provide a real opportunity to provide an integrated telemedicine

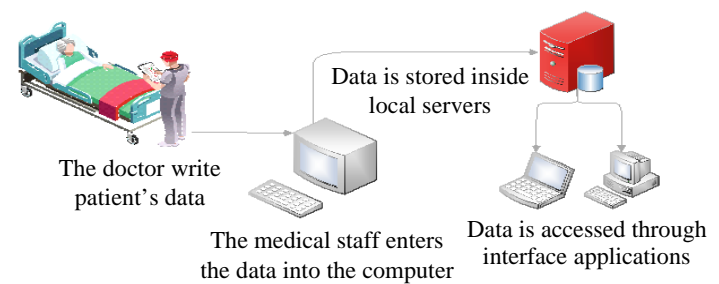

Fig. 1: Depicts the process of writing patient's data

service from the first step of collect patient's data to accessing the information. This system must have the following high-level requirements in it:

The system should implement methods of collecting patient data as well as processing and distributing it from the bed to the possibility of retrieving it remotely. It should be safe, that is the system must ensure the privacy and integrity of patient's data. It should be easy to manage in other words, it must provide control over the huge amount of computers associated with the research environment. It must ensure high confidence, that is the availability of the system must be guaranteed in all different operating conditions. It must be flexible, open and extendable, that is the addition of heterogeneous devices in different numbers to the system must be supported. It must be well prepared for computing resources, that is the system works on cheap and inexpensive computing devices.

\section{Benefits of medical cloud computing}

Provide a better treatment service: The availability of a unified medical record of patients at anytime and anywhere helps doctors to see the patient's medical history which helps to treat him better (Parveen et al., 2013).

Help transform in healthcare services: Cloud computing supports one-team care provision as well as the ability to use applications based on the requirements of a business model. It can be done on a platform that allows health institutions to introduce integrate and use new services based on a comprehensive and patient vision regardless of where or from whom care has been delivered (Anonymous, 2011).

Low cost: Because of the feature of sharing of resources for cloud computing, the cost of building an infrastructure of IT is reduced, so that, the client only needs to match the minimum cost of the common infrastructure with the flexibility to pay for the use of actual resources only.

The quality improve: Since, all medical data is stored in one place, it will be easy to provide it to the Ministry of Health or WHO through patient safety and the quality of treatment provided. 
Support scientific research: As we have stated that all data are available in one place, it will be easy to conduct medical research to find new medical facts, thereby improving the treatment services provided to patients.

Easy access to patient's data from anywhere: The possibility of using the cloud facilities without need the presence of the actual doctor or medical staff in the health institutions (Bhatia et al., 2013).

Support decision making: Medical cloud computing data can be used by decision-makers in health institutions to plan and budget for health care services (AbuKhousa et al., 2012).

\section{RESULTS AND DISCUSSION}

Proposed system: The proposed system for solving problems facing medical staff in hospitals depends on cloud computing technology where all data collected from patients is stored in the cloud rather than on the local servers. Therefore, it is easy to access this data through any electronic device connected to the internet, whether personal computer or smart mobile or tablet at any time and from anywhere.

Figure 2 depicts the proposed structure of the system. Where the doctor writes a patient's data using the tablet/smartphone that connected to the internet through a graphical user interface and directly this data uploaded to the cloud system for storing. Then, this data is available to the medical staff whose use this medical cloud. Medical staff uses internet-connected devices such as tablets, personal computers or smartphones. These devices in addition to internet access are equipped with an internet browser that enables them to access the medical cloud and obtain vital data for patients in a short time and from any place where they are located, both inside or outside the health institution. Which offers the possibility of telemedicine. There are many practical benefits of applying this proposed medical cloud:

- Always provides real-time data collection

- Eliminates manual data collection which reduces the possibility of typographical errors

- Access to patient data requires only a web-connected device with a browser and therefore does not require special systems that need periodic maintenance

- Connecting devices to the internet service is wireless and therefore does not require cables or any other physical configuration

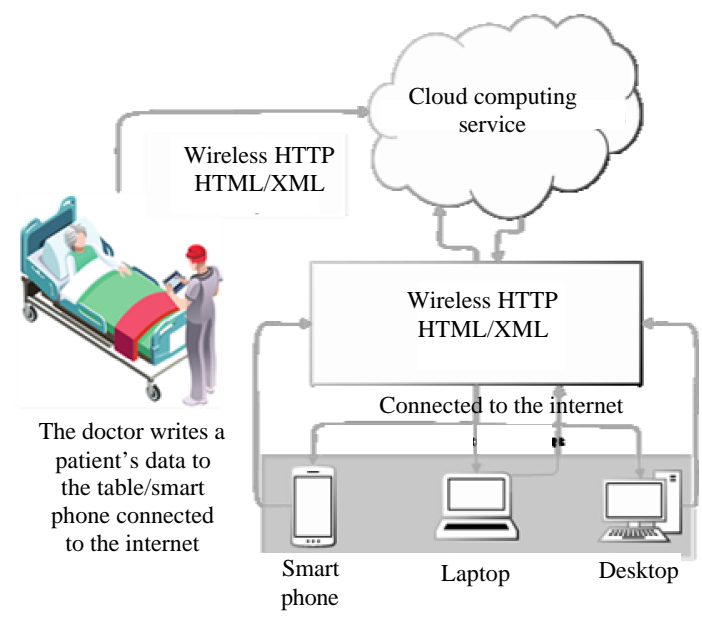

Fig. 2: Proposed system

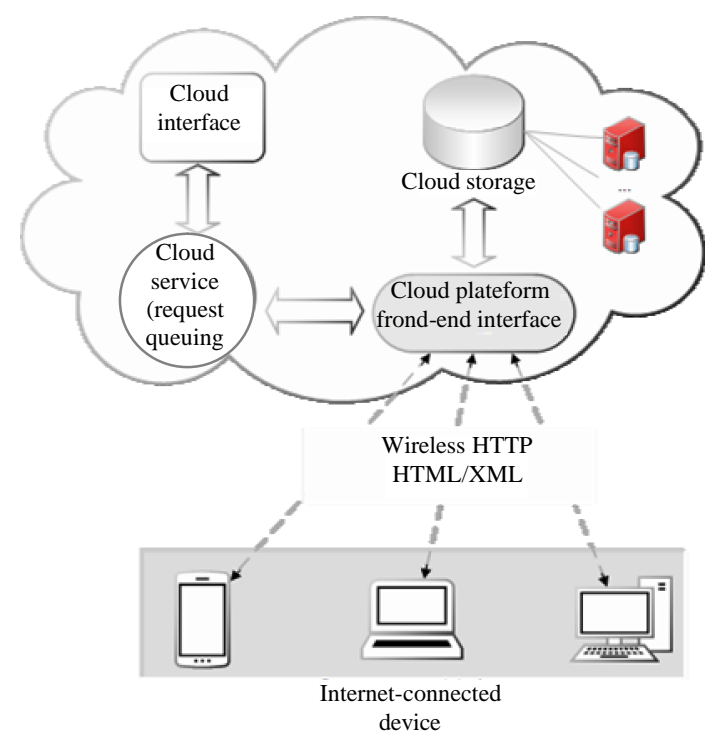

Fig. 3: The architecture of the proposed system

Architecture of the proposed system: Figure 3 illustrates the architecture of the proposed system for deploying healthcare systems in Iraqi Health Institutions that utilizing cloud computing. The main part of the cloud computing service is the front-end interface of the platform which directly connects users who're using (internet-connected devices) and allows management of cloud storage content. The cloud platform interface can be a web client or a web application. On the other hand, the cloud storage manages storage elements that represent the physical storage infrastructure, also responsible for data maintenance (for example, data backup). The cloud platform is also linked to a cloud service that handles and queues user's requests in 
queues. Finally, the cloud infrastructure manages user accounts for the medical cloud, cloud access and the authority of each user from a medical staff.

\section{CONCLUSION}

In short, our proposed system provides practical solutions to the problems faced by doctors and medical staff in Iraqi Health Institutions. Where, there are many practical advantages provided by this cloud-based system, for example, it always provides real-time data collection, it eliminates manual data collection which reduces the possibility of typographical errors in addition to the accessibility of medical staff to the vital data of patients at any time and from any place where doctors are located. So, it providing the possibility of telemedicine service. The implementation of this proposed system will reduce the time and effort required to provide health services to patients which helps doctors and supports them in the speed of decision-making, resulting in the rapid reception of patients for treatment which is the main objective of this proposed system.

\section{RECOMMENDATIONS}

For future researches, we intend to implement this proposed system in a health institution to verify the validity of the proposal in the real world and evaluate the benefits gained from this system on a large scale. It will also add some improvements regarding the management and information security. In addition to strengthening the system with the ability to exchange medical images which has become vital information that help doctors in the diagnosis of diseases.

\section{REFERENCES}

AbuKhousa, E., N. Mohamed and J. Al-Jaroodi, 2012. E-Health cloud: Opportunities and challenges. Future Internet, 4: 621-645.

Anonymous, 2011. Cloud computing: Building a new foundation for healthcare. IBM Computer Hardware Company, New York, USA.

Aziz, H.A. and A. Guled, 2016. Cloud computing and healthcare services. J. Biosens. Bioelectron., 7: $1-4$.

Bamiah, M., Brohi, S. and S. Chuprat, 2012. A study on significance of adopting cloud computing paradigm in healthcare sector. Proceedings of the 2012 International Conference on Cloud Computing Technologies, Applications and Management (ICCCTAM), December 8-10, 2012, IEEE, Dubai, United Arab, ISBN:978-1-4673-4415-9, pp: 65-68.
Bhatia, G., A. Lala, A. Chaurasia and R. Rajpal, 2013. Implementation of cloud computing technology for the improvement of entire healthcare services in India. Proceedings of the 2013 International Conference on Advances in Technology and Engineering (ICATE), January 23-25, 2013, IEEE, Mumbai, India, ISBN:978-1-4673-5618-3, pp: $1-5$.

Daman, R., M.M. Tripathi and S.K. Mishra, 2016. Security issues in cloud computing for healthcare. Proceedings of the 20163 rd International Conference on Computing for Sustainable Global Development (INDIACom), March 16-18, 2016, IEEE, New Delhi, India, ISBN:978-1-4673-9417-8, pp: 1231-1236.

Doukas, C., T. Pliakas and I. Maglogiannis, 2010. Mobile healthcare information management utilizing cloud computing and android OS. Proceedings of the Annual IEEE International Conference on Engineering in Medicine and Biology Society (EMBS), August 31- September 4, 2010, IEEE, Buenos Aires, Argentina, ISBN:978-1-4244-4123-5, pp: 1037-1040.

Huang, Q., L. Ye, M. Yu, F. Wu and R. Liang, 2011. Medical information integration based cloud computing. Proceedings of the 2011 International Conference on Network Computing and Information Security (NCIS), May 14-15, 2011, IEEE, Guilin, China, ISBN:978-1-61284-347-6, pp: 79-83.

Kanagaraj, G. and A.C. Sumathi, 2011. Proposal of an open-source cloud computing system for exchanging medical images of a hospital information system. Proceedings of the 2011 3rd International Conference on Trendz in Information Sciences and Computing (TISC), December 8-9, 2011, IEEE, Chennai, India, ISBN: 978-1-4673-0134-3, pp: 144-149.

Mei, R. and X. Qiu, 2016. General medical information management system design based on cloud computing. Proceedings of the 2016 International Conference on Network and Information Systems for Computers (ICNISC), April 15-17, 2016, IEEE, Wuhan, China, ISBN:978-1-4673-8838-2, pp: 265-268.

Parveen, A., S. Habib and W. Ahmad, 2013. The cloud-changing the Indian Healthcare System. Intl. J. Comput. Sci. Mob. Comput., 2: 238-243. 
Rolim, C.O., F.L. Koch, C.B. Westphall, J. Werner and A. Fracalossi et al., 2010. A cloud computing solution for patients data collection in health care institutions. Proceedings of the 2010 2nd International Conference on eHealth, Telemedicine and Social Medicine, February 10-16, 2010, IEEE, St. Maarten, Netherlands Antilles, ISBN:978-1-4244-5803-5, pp: 95-99.
Tejaswi, A., N.M. Kumar, G. Radhika and S. Velagapudi, 2012. Efficient use of cloud computing in medical science. Am. J. Comput. Math., 2: 240-243.

Wang, L. and C.A. Alexander, 2013. Medical applications and healthcare based on cloud computing. Intl. J. Cloud Comput. Serv. Sci., 2: 217-225. 